\title{
P83 Effect of Aging on the Aortic Reservoir and Excess Pressure in Normal Humans
}

\author{
Tetsu Yamakado*, Hideyoshi Ko \\ Suzuka University of Medical Science, Suzuka City, Mie, Japan
}

\section{ABSTRACT}

Background: Reservoir pressure analysis has been newly introduced to analyze arterial waveform and function. There are few data regarding the effect of aging on the aortic reservoir and excess pressures in normal humans.

Objectives and Methods: To investigate this issue, we analyzed high-fidelity aortic pressures, measured by a catheter-tipped manometer in 81 normal subjects who underwent diagnostic cardiac catheterization but proved to have normal coronary artery and LV function. Subjects who had hypertension, diabetes, renal disease or dyslipidemia were excluded. There were 57 men and 24 women, ranging in age from 21 to 80 years. We analyzed the entire AoP and calculated the reservoir pressure (Pr) and the excess pressure (Pex) according to the method by Aguado-Sierra et al., and augmented pressure (AP) was obtained as well.

Results: Systolic AoP and pulse pressure (PP) were significantly increased with age. Pr (amplitude) and Pex (amplitude) were $38.2 \pm 6.3 \mathrm{mmHg}$ and $17.8 \pm 4.2 \mathrm{mmHg}$, respectively. Both $\operatorname{Pr}$ and Pex showed significant age-related change (Pr: $r=0.40$, $p<0.001$, Pex: $r=0.26, p<0.05)$. Age-related increase in PP or AP was more strongly correlated with the $\operatorname{Pr}(r=0.88$ and 0.90 both $p<0.001)$ than the Pex $(r=0.56$ and 0.35 , both $p<0.001)$.

Conclusion: We documented the aortic Pr and Pex in normal humans. The Pr and Pex were both roughly but significantly correlated with aging at least until the ninth decade, while the Pr contributes more to age-related change in PP and AP than the Pex. These data may provide reference data about an arterial function in normal and pathological conditions.

(C) 2019 Association for Research into Arterial Structure and Physiology. Publishing services by Atlantis Press International B.V. This is an open access article distributed under the CC BY-NC 4.0 license (http://creativecommons.org/licenses/by-nc/4.0/). 